\title{
Integration of Radar Measurements with Navigation Data for Initial Orbit Estimation
}

\author{
Nanke Du, 2, a , Linyang Guo ${ }^{3, b}$, Junshan $\mathrm{Mu}^{1, \mathrm{c}}$, Xuwei Su${ }^{1,2, \mathrm{~d}}$ \\ ${ }^{1}$ China Satellite Maritime Tracking and Controlling Department, Jiangyin 214431, China; \\ ${ }^{2}$ Yuanwang 3 Tracking Ship, Jiangyin 214431, China; \\ ${ }^{3}$ CNGC North Laser Technology Group Co.LTD, Yangzhou 225000,China. \\ a moctiors@foxmail.com ,bchirs0232@sina.com , \\ c mujunshan@gmail.com ,d1649666197@qq.com
}

Keywords: Data fusion ; finite difference ; Radar measurements ; orbit determination precision

Abstract:

An introduction is given about the current initial orbit determination mode for tracking ships. The paper proposes a method to improve the current orbit estimation mode by integrating the radar tracking measurements and onboard navigation data. The measurement and system model for the method is given, and related state transition matrix formulation is analyzed. The method is based on Markov and random process theory. The numerical results show that the finite difference method can be a good alternative for the traditional orbit estimation method.

\section{Introduction}

Improving launch vehicle stateestimation accuracy is the ultimate goal of data tracking in the instrumentation ships. To improve the precision of orbit determination, it can start from two approaches: one is to improve the measurement precision of ranging equipment, another is to improve the accuracy of processing methods. The improvement of the precision of equipment usually will lead to a considerable amount of funds for device refurbishement. Improving the processing methods will be more economical and generate higher return.

In this paper, a sequential batch processing mode of statistical orbit determination method for multiple source and multiple phase are proposed. The integration of the radar tracking and onboard navigation data before and after the injection of a spacecraft is modeled and tested. The basic principle and the idea are as follows: Since the process of the satellite separation is a determined Markov process which can be fully described and determined. Based on the theory about Markov process and Bayes' theorem, the integration of data from the multiple phase and source can make the estimation process more stable and accurate.

\section{The measurement Model}

In the phase of spacecraft injection, there are at least radar tacking measurements and onboard navigation data which can be used for the initial orbit determination of the spacecraft. The measurement by radar includes ranging, velocity and angle, etc. Onboard navigation data include pseudo ranging and process position information.

$$
\begin{aligned}
& r=\sqrt{x_{r}^{2}+y_{r}^{2}+z_{r}^{2}} . \\
& \stackrel{\text { uf }}{\rho}=\left(\begin{array}{lll}
x_{s}-r & \cos \lambda & \cos \theta \\
y_{s}-r & \cos \lambda & \cos \theta \\
z_{s}-r & \cos \lambda & \cos \theta
\end{array}\right) \text {. }
\end{aligned}
$$




$$
\begin{aligned}
& \left(\begin{array}{l}
\rho_{u} \\
\rho_{e} \\
\rho_{n}
\end{array}\right)=\left(\begin{array}{ccc}
\cos \lambda & 0 & \sin \lambda \\
0 & 1 & 1 \\
-\sin \lambda & 0 & \cos \lambda
\end{array}\right)\left(\begin{array}{ccc}
\cos \theta & \sin \theta & 0 \\
-\sin \theta & \cos \theta & 0 \\
0 & 0 & 1
\end{array}\right) \rho . \\
& \alpha=\arctan \left(\frac{\rho_{e}}{\rho_{n}}\right) . \\
& \beta=\arctan \left(\frac{\rho_{u}}{\sqrt{\rho_{u}^{2}+\rho_{e}^{2}}}\right) .
\end{aligned}
$$

where $\rho$ is the range vector in the inertial geocentric system, $\lambda$ is the latitude of the sensor, $\theta$ is the sidereal time of the sensor and $(x, y, z)_{s}$ are its geocentric coordinate. Two coordinates can be translated mutually. $\rho_{u}, \rho_{e}, \rho_{n}$ are the sensor station coordinate which are in up, east and north direction.

The elementary data provided by a GPS receiver comprise code and carrier-phase measurements that are obtained by synchronizing the incoming signal with a receiver generated replica [1]. A GPS receiver can receive signal from GPS satellite in either or both of L1 and L2 frequencies. For dualfrequency receivers, the ionospheric delay can be corrected by using the fact that the delay is essentially proportional to the signal frequency. In this paper, we only consider single frequency receiver case and use pseudo range data. The pseudo range between GPS receiver with a GPS satellite is

$$
\begin{aligned}
& \rho=c\left(t_{t}-t_{r}+\delta t_{r}\right)+\delta \rho_{e} . \\
& \rho=\sqrt{\left(x_{i}-x_{r}\right)^{2}+\left(y_{i}-y_{r}\right)^{2}+\left(z_{i}-z_{r}\right)^{2}}+c \delta t_{r}+\delta \rho_{e} .
\end{aligned}
$$

where $\rho$ is the pseudo range, $t_{t}$ is the time of a message transmission from a GPS satellite, $t_{r}$ is the reception time of that message, $c$ is the speed of light, $\delta t_{r}$ is the receiver clock bias, $\delta \rho_{e}$ contains effects of additional error sources such as the ionospheric delay and multipath effect, $(x, y, z)_{i}$ are the coordinates of a GPS satellite in a geocentric coordinate frame and $(x, y, z)_{r}$ are the coordinate of the GPS receiver installed on the last stage of a launch vehicle which, as we have assumed, is in the same position as that of the transceiver of the spacecraft. While the GPS satellite clock offset can be assumed to be known with an adequate accuracy from pre-computed GPS ephemeris and clock products, the receiver clock offset $c \delta t_{r}$ is unknown and has to be determined at each epoch as part of the orbit determination process [7]. The clock bias can be set as an unknown constant in the short arc orbit determination problem.

\section{Orbit Estimation Model and State Transit Matrix Approximation}

\section{A. System Model}

Assume that the epoch at the start of the payload separation from its carrier is $T_{f}^{-}$and the relevant state vector is $\mathbf{X}_{f}^{-}$, where $\mathbf{X}_{f}^{-}=\left[x_{s}, y_{s}, z_{s}, \underset{s}{\alpha}, \underset{s}{\alpha}\right]^{T}$. The separation process is very short, it can be regarded as an impulse process. The process will let the spacecraft obtain a velocity increment $\mathbf{V}_{s}$, so that the velocity of the satellite at the moment after the injection can be denoted as $\mathbf{X}_{f}^{+}$, where 
$\mathbf{X}_{f}^{+}=\mathbf{X}_{f}^{-}+\left[\begin{array}{ll}\mathbf{0} & \mathbf{V}_{s}\end{array}\right]^{T}$. The launch vehicle will obtained a decrement in velocity $\mathbf{V}_{r}$ with oppiste sign with $\mathbf{V}_{s}$

$$
\mathbf{V}_{r}=-\frac{m_{s}}{m_{r}} \mathbf{V}_{s}
$$

where $m_{s}$ is the mass of the spacecraft, $m_{r}$ is the mass of the launch vehicle.

The state of the launch vehicle at the instant after the injection is denoted as $\mathbf{X}_{r}$, where $\mathbf{X}_{r}=\mathbf{X}_{f}^{-}+\left[\begin{array}{ll}\mathbf{0} & \mathbf{V}_{r}\end{array}\right]^{T}$. Here, $\mathbf{X}_{f}^{-}$is used as the parameter to be estimated. The parameters, $\mathbf{V}_{s}, \mathbf{V}_{r}, \mathbf{X}_{r}$, $\mathbf{X}_{f}^{+}$can be obtained through other channels.

The measurements available at the injection can be divided into three segements. The first is the segment before the separation, which starts from the target capturing by tracking radar. The second segement is from the separation to the end of the radar tracking. The third is launch vehich measurement from separation. After the separation, the distance between the two object is very close, the end time of tracking for both objects are basically the same. The traditional methods can handle those segments of data separately and independently.

$$
\begin{aligned}
& \mathbf{X}_{f}=\operatorname{argmin} J_{c}=\arg \min \sum_{t=T_{\text {sot }}}^{T_{f}^{-}}\left(g\left(t, \mathbf{X}_{f}^{-}\right)-\mathbf{Y}_{o c}(t)\right)^{2} . \\
& \mathbf{X}_{f}^{+}=\arg \min J_{s}=\arg \min \sum_{t=T_{f}^{+}}^{T_{\text {sop }}}\left(g\left(t, \mathbf{X}_{f}^{+}\right)-\mathbf{Y}_{o s}(t)\right)^{2} . \\
& \mathbf{X}_{r}=\arg \min J_{r}=\arg \min \sum_{t=T_{f}^{+}}^{T_{\text {sop }}}\left(g\left(t, \mathbf{X}_{r}\right)-\mathbf{Y}_{o r}(t)\right)^{2} .
\end{aligned}
$$

where, $\mathbf{Y}_{o c}(t), \mathbf{Y}_{o s}(t), \mathbf{Y}_{o r}(t)$ are the measurements observation vectors. It is possible that the elements of the observation at different time will not fully same. There are many elements of measurements in these segments, such as radar angles and ranging elements, navigation data, etc. Here, we proposed to use these measurements jointly, and estimate the initial orbit by the following data integration

$$
\mathbf{X}_{f}^{+}=\arg \min J=\arg \min \left\{J_{c}+J_{s}+J_{r}\right\} .
$$

There are many options for numerical optimization. Newton-Raphson method is common method in orbit estimation problem. These gradient methods for nonlinear problems will inevitably need the formulation and calculation of state transit matrix. There are mainly three types of formulation for state transit matrix used in TT\&C ships. One is traditional differential orbit correction method. The second is finite differencing method. The third is Unit Vector Method $[5,8,9,10]$. The three methods can be used in different situation with varied accuracy, and have their own advantages and disadvantages, respectively.

\section{B. State transit matrix obtained by Unit Vector Method}

The basic principle is also from the differential correction method [6, 7]. Its perturbed conditional equation can be written as

$$
\left\{\begin{array}{l}
\mathbf{r}_{i}=f_{i} \mathbf{r}_{0}+g_{i} \mathbf{\alpha}+w_{i} \mathbf{w}_{0} \\
\mathbf{\phi}=f_{i}^{\prime} \mathbf{r}_{0}+g_{i}^{\prime} \mathbf{\alpha}+w_{i}^{\prime} \mathbf{w}_{0}
\end{array} \quad(i=1,2,3, \mathrm{~L}, n) .\right.
$$


where, $\mathbf{r}_{i}$ is the state vector $\left[x_{s}, y_{s}, z_{s}\right]^{T}$ of target at the $i$ th time step, $\alpha_{i}$ is the velocity vector $\left[\&_{s}, \&_{s}, \&_{s}\right]^{T}$ at the time, $\mathbf{X}_{i}=\left[\begin{array}{l}\mathbf{r}_{i} \\ \boldsymbol{\alpha}_{i}\end{array}\right]$. The derivative of Eq. (13) relative to the state vector $\mathbf{X}_{0}$ at a predetermined epoch can written as

$$
\left\{\begin{array}{l}
d \mathbf{r}_{i}=\delta \mathbf{r}_{i}+\Delta \mathbf{r}_{i} \\
d \mathbf{\alpha}=\delta \underset{l}{\mathbf{\alpha}}+\Delta \mathbf{\alpha}
\end{array}\right.
$$

where

$$
\left\{\begin{array}{l}
\delta \mathbf{r}_{i}=\left(\frac{\partial f_{i}}{\partial \mathbf{X}_{0}} \cdot d \mathbf{X}_{0}\right) \cdot \mathbf{r}_{0}+\left(\frac{\partial g_{i}}{\partial \mathbf{X}_{0}} \cdot d \mathbf{X}_{0}\right) \cdot \boldsymbol{\phi}+\left(\frac{\partial w_{i}}{\partial \mathbf{X}_{0}} \cdot d \mathbf{X}_{0}\right) \cdot \mathbf{w}_{0} \\
\delta \underset{\boldsymbol{\alpha}}{\mathbf{\alpha}}=\left(\frac{\partial f_{i}^{\prime}}{\partial \mathbf{X}_{0}} \cdot d \mathbf{X}_{0}\right) \cdot \mathbf{r}_{0}+\left(\frac{\partial g_{i}^{\prime}}{\partial \mathbf{X}_{0}} \cdot d \mathbf{X}_{0}\right) \cdot \boldsymbol{\phi}+\left(\frac{\partial w_{i}^{\prime}}{\partial \mathbf{X}_{0}} \cdot d \mathbf{X}_{0}\right) \cdot \mathbf{w}_{0}
\end{array} \cdot\right.
$$

and

$$
\left\{\begin{array}{l}
\Delta \mathbf{r}_{i}=f_{i} \cdot d \mathbf{r}_{0}+g_{i} \cdot d \mathbf{\alpha}+w_{i} \cdot \frac{\partial \mathbf{w}_{0}}{\partial \mathbf{X}_{0}} \cdot d \mathbf{X}_{0} \\
\Delta \underset{\mathbf{\alpha}}{\mathbf{\alpha}}=f_{i}^{\prime} \cdot d \mathbf{r}_{0}+g_{i}^{\prime} \cdot d \boldsymbol{\alpha}+w_{i}^{\prime} \cdot \frac{\partial \mathbf{w}_{0}}{\partial \mathbf{X}_{0}} \cdot d \mathbf{X}_{0}
\end{array} .\right.
$$

When the data arc is short and $\left|T_{i}-T_{0}\right|$ is small, $\delta \mathbf{r}_{i}, \delta \underset{q}{\alpha}$ are small quantities compared with $\Delta \mathbf{r}_{i}, \Delta \underset{l}{\alpha}$. Namely, $\delta \mathbf{r}_{i}, \delta \phi$ are trivial items in the formations above. If neglecting the trivial items and main items are kept, the following approximation can be obtained

$$
\left\{\begin{array}{l}
d \mathbf{r}_{i} \approx \Delta \mathbf{r}_{i} \\
d \mathbf{f} \approx \approx \sim \boldsymbol{\alpha}
\end{array} .\right.
$$

When the data arc is short and only main items are used, the following equation can be obtained

$$
\left\{\begin{array}{l}
\Delta \mathbf{r}_{i}=f_{i} \cdot \Delta \mathbf{r}_{0}+g_{i} \cdot \Delta \mathbf{\alpha}+w_{i} \cdot \frac{\partial \mathbf{w}_{0}}{\partial \mathbf{X}_{0}} \cdot \Delta \mathbf{X}_{0} \\
\Delta \underset{i}{\mathbf{\alpha}}=f_{i}^{\prime} \cdot \Delta \mathbf{r}_{0}+g_{i}^{\prime} \cdot \Delta \underset{\alpha}{\alpha}+w_{i}^{\prime} \cdot \frac{\partial \mathbf{w}_{0}}{\partial \mathbf{X}_{0}} \cdot \Delta \mathbf{X}_{0}
\end{array} .\right.
$$

The equation can be simplified as $[2,3,4]$

$$
\Delta \mathbf{X}_{i}=\Phi_{i} \cdot \Delta \mathbf{X}_{0} .
$$

where $\Phi_{i}=\left(\begin{array}{ll}f_{i} \cdot E_{3 \times 3} & g_{i} \cdot E_{3 \times 3} \\ f_{i}^{\prime} \cdot E_{3 \times 3} & g_{i}^{\prime} \cdot E_{3 \times 3}\end{array}\right)+\left(\begin{array}{c}w_{i} \cdot \frac{\partial \mathbf{w}_{0}}{\partial \mathbf{X}_{0}} \\ w_{i}^{\prime} \cdot \frac{\partial \mathbf{w}_{0}}{\partial \mathbf{X}_{0}}\end{array}\right)$.

\section{State transit matrix by Finite difference}


The goal of the differential correction is obtain the error state transit matrix $\frac{\partial \mathbf{X}_{i}}{\partial \mathbf{X}_{0}}$ and $\frac{\partial \mathbf{Y}_{i}}{\partial \mathbf{X}_{0}}$, which are the derivative of state vector $\mathbf{X}_{i}$ and observation vector $\mathbf{Y}_{i}$ at the time $T_{i}$ relative to the state $\mathbf{X}_{0}$ at the time $T_{0}$. The Jocobian matrix $\frac{\partial \mathbf{X}_{i}}{\partial \mathbf{X}_{0}}$ can be expressed as

$$
\frac{\partial \mathbf{X}_{i}}{\partial \mathbf{X}_{0}}=\left(\begin{array}{ll}
\frac{\partial \mathbf{r}_{i}}{\partial \mathbf{r}_{0}} & \frac{\partial \mathbf{r}_{i}}{\partial \hat{q}} \\
\frac{\partial \hat{q}}{\partial \mathbf{r}_{0}} & \frac{\partial \dot{q}}{\partial \boldsymbol{q}}
\end{array}\right)
$$

The idea behind the finite difference is simple. That is to give an increase $\Delta \mathbf{X}_{i}$ in one direction of initial vector, and the equation above can be approximated as

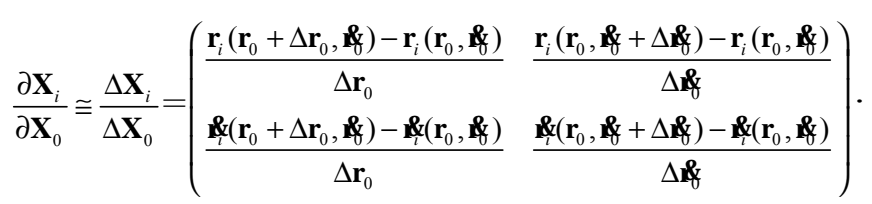

\section{Numerical Results}

In order to isolate the random distribution of uncertainty appeared in actual measurement data of past missions, the paper uses simulated data to test the proposed method. In the paper, the orbit for a historical mission is used as reference orbit, and STK is used to propagate the reference orbit. Simulated tracking measurements with designed noise are obtained through the use of simulated ship positions. Simulated navigation data with noise are also simulated based on the reference ephemeris. In orbit calculation process, the measure data are categorized into three groups: the first group of data are the measurements for the combined body before the separation which include radar tracking and GPS pseudo ranging measurements. The second group include the measurements for the combined body before the separation and the measurements for the launch vehicle after the separation, which include radar tracking and GPS pseudo ranging measurements. The third group data contains the measurements for the combined body before the separation, the measurements for the launch vehicle after the separation and the measurements for the spacecraft after the separation.

The two methods discussed above are used to test the three groups of data. The orbit determination results show that

(1) When the same method is used, the result generated by the second groups and third groups are significantly better than those generated by the first group.

(2) The results generated by the third group are slightly better than the results generated by the second group. The difference is not obvious, this phenomenon is consistent with the relative lower precision of radar tracking.

(3) The results generated by the finite difference method for the same set of data are more optimal the results generated the Unit Vector Method.

\section{Conclusion}

The paper proposed to integrate the radar tracking measurements and onboard navigation data before and after injection. The method has its following advantages:

(1) Current method processes the measurements separately and independently. However, the proposed one can handle multiple phase and sources concurrently.

(2) Data range used by current orbit determination mode is very short. It can result in lower stability and accuracy in the estimation process. If Unit Vector Method is applied, it has larger region of 
convergence. However, it has lower accuracy. Thus, finite difference is a good and easy option the initial orbit determination for short arc, multiple source and multiple phase.

\section{References}

[1] P. Misra and P. Enge, Global positioning system : signals, measurements, and performance, 2nd ed. ed. Lincoln, Mass.: Ganga-Jamuna Press, 2006.

[2] O. Montenbruck and G. Eberhard, Satellite orbits : models, methods, and applications. Berlin ; London: Springer, 2000.

[3] B. D. Tapley, et al., Statistical orbit determination. Amsterdam ; London: Elsevier Academic Press, 2004.

[4] D. A. Vallado, Fundamentals of astrodynamics and applications, 3rd. ed. New York: Springer, 2007.

[5] Chen Wusheng, et al, "The convergence prerformance of MMUVM and PUVM1," Astronomy Journal, vol. 48, pp. 343-354, 2007(In Chinese).

[6] Li Jisheng, Precise Orbit Determiniation for Man-made satellite. Beijing: PLA Publishing House, 1995(In Chinese).

[7] Liu Lin, The Orbit Theory of Spacescraft. Beijing: National Defense Publishing House, 2000(In Chinese).

[8] J. Zhang, et al., " A new method for orbit determination: Unit vector method, Science in China Series G: Physics," Science in China Series G: Physics, Mechanics Astronomy, vol. 52, pp. 1115-1119, 2007.

[9] Lu Benkui,et al, "A new perturbed unit vector method," Astronomy Journal, vol. 20, pp. 14-22, 1999(In Chinese).

[10]Zhang Yuxiang, Satellite Orbit Determination. Beijing: National Defence Publishing House, 2007(In Chinese). 